\title{
We need more regional heart failure clinics in Slovakia in real life
}

\author{
Alexander Klabnik ${ }^{1 *}$, Jan Murin ${ }^{2}$ \\ ${ }^{1}$ Hospital Banovce, Banovce nad Bebravou, Slovak Republic \\ ${ }^{2}$ University Hospital, Bratislava, Slovak Republic
}

Purpose: At this time we have only three active heart failure (HF) clinics in the metropolitan areas of Slovakia and no organised HF disease management programme exists in rural, regional settings.

Patients and Methods: We compared clinical characteristics, evidence-based medications and outcomes in 118 patients (pts) from one regional centre with other centres in Slovakia $(n=306$ ) that participated in the Heart Failure Long-Term registry. We statistically analysed the reasons for differences in the benchmark report.

Results: In the regional centre, the patients significantly ( $p$ $<0.05)$ older than $65(74.6 \%$ vs $32.7 \%$, odds ratio, OR $>5)$, with non-ischemic etiology $(71.2 \%$ vs $41.7 \%$, OR 3.46 , predominantly valvular etiology), more advanced HF (in NYHA class III-IV: $61 \%$ vs $30.1 \%$, OR 3.63) and fewer males (57.6\% vs $70.5 \%$, OR 0.57 ) were recruited as outpatients. Common comorbidities were significantly $(p<0.05)$ more

Received: $1^{\text {st }}$ May 2014

*Address for correspondence: Hospital Banovce, Hviezdoslavova 23/3, 95701 Banovce nad Bebravou, Slovak Republic.

Phone: +424907070432

E-mail: sanus@zoznam.sk often present in the regional centre: diabetes $(44.1 \%$ vs $25 \%$, OR 2.36), chronic renal dysfunction (35.6\% vs $10.3 \%$, OR 4.84$)$, current malignant cancer $(15.3 \%$ vs $1.3 \%$, OR $>5)$, permanent atrial fibrillation $(45.8 \%$ vs $8.3 \%$, OR $>5)$. At the first glance, it seemed that ACE-inhibitors/sartans (71.2\% vs $90.4 \%$, OR 0.26$)$, beta-blockers (78\% vs $90.4 \%$, OR 0.38$)$ and mineralocorticoid receptor antagonists $(23.7 \%$ vs $60.9 \%$, OR 0.20$)$ are significantly $(p<0.05)$ underused in the regional centre. But after considering the objective reasons for non-using these medications in the regional centre, these differences disappeared. In regional centre, more pts have a history of previous hospitalisation $(64.4 \%$ vs $28.6 \%$, OR 4.52). During a 12 month follow-up more pts in the regional centre died $(20.3 \%$ vs $4.4 \%$, OR $>5$ ) and were more often re-hospitalised: 1 st $(66.1 \%$ vs $16.4 \%$, OR $>5)$, respectively the 2 nd re-hospitalisation ( $48.7 \%$ vs $11.8 \%$, OR $>5$ ). The hazard of death and re-hospitalisation increased with more advanced age and multiple comorbidities.

Conclusion: In Slovakia, HF clinics are urgently needed in the regional settings in order to improve the quality of HF care and optimized outcomes.

KEYWORDS: heart failure, disease management, regional differences, quality of care.

CITATION: Cardiol Croat. 2014;9(5-6):221 